tonsil. If sclerosis of the tympanum, or if a previous suppuration within the tympanum were associated with these synechiæ, the mere treatment of the latter would not affect a great improvement, but as the diagnosis of any case was narrowed down to the existence of mere tubal catarrh, it could be safely stated that the treatment of these synechiæ would immensely favour the desired result. For the purpose of destroying these bands the galvano-cautery was impracticable and useless. A much more extensive destruction was necessary. The finger was the best means to effect this, carried up to the bands, which were readily felt. The resistance to rupture was often considerable. The finger was carried round the tube, so as to rupture not only those attached superiorly, but also those extending from bands of later granular pharyngitis inferiorly. There was often considerable bleeding, which of itself often acted beneficially on the ear. Subsequently the periphery of the tube was daily swabbed with a 4 per cent. solution of nitrate of silver to remove the collateral hyperæmia of the mucous membrane which was present. The air douche with catheter or Politzer's bag was, of course, to be used. When only tubal catarrh had been the cause then recovery was rapid and satisfactory, but even in more advanced and chronic conditions with the tympanum the same treatment must be carried out.

Dr. Mirligan (Manchester) congratulated Dr. Robertson on so clearly drawing attention to the relation between intranasal and aural conditions. Due attention to the condition of both the anterior and posterior nares in all aural conditions was of paramount importance. He agreed with Dr. Robertson as to the etiology of the production of Eustachian synechiæ, and thought that the best plan of destroying them was to scrape them away with the finger nail.

Dr. BRonNer (Bradford) was glad to see that Dr. Robertson had again drawn attention to the intimate relations between the diseases of the nose, the naso-pharynx, and the middle ear. Swelling and synechiæ near the Eustachian prominence were fairly common, and due in many cases to hypertrophy of the adenoid tissue situated there. The best method of treatment was to remove the synechiæ and swelling by the finger nail or curette. Many cases of middle-ear disease were caused by the injudicious use of the nasal douche in cases of disease of the nares.

Mr. HUGH E. JoNes (Liverpool) thought that deviation of the nasal septum, apart from its more direct effect, often kept up middle-ear inflammation in an indirect way by interference with treatment. Where there was any secretion in the naso-pharynx it was better to avoid, if possible, the use of Politzer's method of inflation, as by it the objectionable secretions must almost inevitably be forced into the Eustachian tube. When the Eustachian catheter could not for this reason alone be passed, it was necessary to excise the projecting portion of the septum. In illustration of this he mentioned a case with naso-pharyngeal catarrh and mucous catarrh of the tympanum, in which catheterism was impossible owing to extreme deviation of the septum. After excision of the most prominent portion of the septum catheterism became possible, the mucous discharge from the tympanum soon stopped, and the hearing improved from W. nil to W. 18".

\section{A NEW INTRATYMPANIC SYRINGE.}

Dr. William Milligan (Manchester) said that in cases of suppurative disease of the middle ear the first indication was to cleanse the tympanic mucous membrane in such a way that local applications might be applied directly to the diseased surface. The difficulties of thoroughly cleansing the parts were, however, at times very great. This was more especially the case when the accompanying perforation was small or when it was situated high up upon the surface of the membrana tympani, for example, when the perforation was in the membrana flaccida Shrapnelli. In such cases the ordinary procedure of syringing along the external auditory meatus was insufficient. Hardly any of the fluid found its way through the small perforation into the cavity of the middle ear to flush the diseased mucous membrane. Washing out the middle ear by way of the Eustachian tube was no doubt a satisfactory method in many cases, but unfortunately many patients resented the passing of the Eustachian catheter. Intratympanic syringes for passing along the external auditory meatus and through the perforation into the cavity of the middle ear had been designed by various aural surgeons, amongst others by Hartmann, Blake, Politzer, and Pritchard, and had been found of much service. These syringes were, however, somewhat difficult to manipulate, and from the position in which they had to be held, partially obstructed the view of the deeply-lying parts of the ear. In addition, it was difficult to steady the instrument while at the same time the process of syringing was taking place. These difficulties were in a measure overcome by using an intratympanic syringe fed from an elevated reservoir. The advantages of this form of syringe were (1) that it could be held perfectly steady while the fluid was streaming from the reservoir into. the middle ear; $(2)$ that during the whole manipulation the point of the syringe was kept constantly in view ; (3) that by altering the weight of the reservoir the pressure of the outgoing fluid could be regulated according to the special requirements of any particular case.

\section{A CASE OF FOREIGN BODY IN THE MASTOID CELLS.}

Dr. Walton 'Browne (Belfast) related a case in which two prongs of a fork two inches long penetrated in front of the tragus, and passed downwards and backwards into the mastoid cells. The prongs were lodged for nineteen months, and caused no serious symptoms. They were removed successfully.

\section{REPORTS} TO THE

SCIENTIFIC GRANTS COMMITTEE OF THE

\section{BRITISH MEDICAL ASSOCIATION.}

\section{REPORT ON \\ IMMUNITY AGAINST “CHOLERA."}

\section{AN EXPERIMENTAL INQUIRY INTO THE BEARING ON IMMUNITY OF "INTRACELLULAR" AND \\ "METABOLIC" BACTERIAL POISONS.}

BY A. A. KANTHACK, M.D., M.R.C.P., Medical Tutor Liverpool Royal Infirmary, AND

F. F. WESBROOK, M.D.,

John Lucas Walker Student in Pathology Cambridge University.

Is a recent paper, Dr. Klein ${ }^{1}$ has opened up a new questiorr with regard to bacterial poisons, a question which before him was partially already touched upon by $\mathbf{R}$. Pfeiffer. ${ }^{2}$ Klein showed that when emulsions of various cultures grown on agar agar, prepared in the same manner as that employed by Haffkine ${ }^{3}$ in his "anticholeraic vaccinations," are injected into the peritoneal cavity of guinea-pigs, the result is the same as that following on a similar injection of an emulsion of an agar-agar culture of cholera bacilli. Klein used cultures of cholera bacilli, of the vibrio of Finkler, the bacillus coli, proteus vulgaris, the bacillus prodigiosus, and the bacillus of typhoid fever. By means of intraperitoneal injections of non-fatal doses, he rendered the animals refractory against further intraperitoneal injections of fatal doses, and he found that "the refractory condition produced by intraperitoneal injections of a non-fatal dose of one of the above six species holds good against all the other five." $\mathrm{He}$ argues from his experiments that while employing agar-agar. culture in this manner, the bacilli are used without their specific poisons, and that the pathological changes produced. 
in the animals under such conditions are due to the "protoplasmic" or "intracellular" poison of the micro-organisms; and that probably the "intracellular" poison of these different species of bacteria is identical, since they mutually protect the animals. He further contends that a notable difference exists between the "intracellular" poison and the toxins elaborated by the bacilli in the culture medium, and concludes that though protected against such "intracellular" poison by such methods of vaccination as practised by Haffkine, the animals are not necessarily protected against the toxins elaborated in the culture medium (for example gelatine) by the bacilli. In proof of this, he mentions that guinea-pigs which have survived the intraperitoneal injection of fatal doses of Haffkine's virus fort, when subsequently inoculated intraperitoneally with old liquefied gelatine cultures of cnolera bacilli promptly succumb.

Hitherto it was generally believed that an immunity artificially produced against the bacillus itself was sufficient to protect the animal against the specific infection produced by such bacillus, and so far as immunity is concerned, no distinction was drawn between the "intracellular" and the "metabolic" poisons. Klein's researches suggested so many new questions of vital importance, that it seemed inviting to examine this matter more fully, and to investigate whether an immunity acquired against the "intracellular" poison will also be efficacious against a true specific infection with the same bacillus.

$A$ priori it would seem sufficient to protect the animal in such a manner as to enable the organism to destroy the bacillus itself, thus giving the latter no time or possibility to elaborate its toxins, and no doubt for all practical purposes where preventive inoculation is likely to come into play, this would be all that is required. It is well known that in some cases it is difficult, in others even impossible, to immunise an animal against the specific toxin, administered in lethal doses, although small doses of such toxin introduced by subcutaneous or intraperitoneal injections will readily render the animal refractory against the microbe manufacturing the toxin. This is so well known that a few examples only need be mentioned here, ${ }^{4}$ Gamaleia has observed that guinea-pigs immunised against the vibrio Metschnikovi remain as sensitive to the toxin of this vibrio as non-immunised animals. ${ }^{5}$ Charrin and Gamaleïa further have shown that this is true also for the bacillus pyocyaneus and its toxin ${ }^{6}$ and Selander ${ }^{7}$ and Metschnikoff ${ }^{8}$ have confirmed the same for the microbe of hog cholera and its toxin. Issaeff, 9 lastly, has demonstrated that rabbits immunised against infection with the diplococcus pneumoniæ are, nevertheless, extremely sensitive against the toxin of this microbe.

It is thus evident that the acquired immunity against certain bacteria is not necessarily accompanied or followed by a resistance against the specific poisons of such bacteria, although these poisons were employed to establish the immunity. These facts may explain Klein's remarkable result that animals resistant against large doses of highly virulent comma bacilli succumbed to large doses of old liquefied gelatine cultures, since it is quite possible that the gelatine cultures used by him contained lethal doses of the specific poison in addition to the bacilli present.

We have repeated some of Klein's experiments, and extended them in various directions, and have obtained results which may throw some fresh light on the important question of the relation of immunity to the "intracellular" specific poisons. As might have been expected, we have been able to confirm most of Klein's results.

(1) In our own experiments we have employed the bacillus prodigiosus, bacillus pyocyaneus, and the vibrio of cholera Agar emulsions of any one of them injected into the peritoneal cavity of guinea-pigs proved fatal, the appearances being identical with those described by Klein. Whatever microbe was used for intraperitoneal injections, it was found that in all the fatal cases the bacilli had multiplied enormously in the peritoneal cavity, were almost invariably present in the heart's blood, and, when looked for. also found in the pleural cavity. ${ }^{10}$ This shows that death in all cases was due to a true infection and not an intoxication, as Pfeiffer ${ }^{\text {11 }}$ assumed. The multiplication of the proliferation are a sine quâ non, and are especially striking when small doses of agar emulsions are used. In regard to cholera infections, Gruber and Wiener ${ }^{12}$ have already pointed this out. A similar proliferation also invariably followed on fatal intramuscular or subcutaneous inoculations of the agar emulsions. On the strength of these facts it seems at least doubtful that the fatal lesions should be the result of poisoning by "bacterial proteins." It is more reasonable to assume that we are dealing here with a true infection.

(2) According to Klein, sterilised agar emulsions intraperitoneally injected produce the same result as the agar emulsions, namely, peritonitis and death. As far as cholera is concerned, R. Pfeiffer ${ }^{13}$ agrees with him. Gruber and Wiener, ${ }^{14}$ on the other hand, disagree, and come to the conclusion that the "protoplasmic" poison of the vibrio has nothing to do with the typical infection. Our own experiments in this direction were limited, but we have failed to produce fatal results with sterilised emulsions prepared from half an agar culture of bacillus pyocyaneus and vibrio of cholera, although one-fifth of living agar emulsions sufficed to kill a guinea-pig. We have not followed up this matter, as it lay somewhat outside the purpose of this research, and we are thus unable to reconcile the contradictory statements of Klein and Pfeiffer on the one hand and of Gruber and Wiener on the other.

(3) As might have been expected from Klein's results, we found that intraperitoneal injections of an emulsion of an agar-agar culture of the bacillus prodigiosus prepared in the same manner as that employed by Haffkine in his inoculations will immunise rabbits and guinea-pigs without fail against similar inoculations with agar cultures of the bacillus pyocyaneus. It is immaterial whether the immunising emulsion is given intraperitoneally or subcutaneously, or whether it is living or sterilised; the result is the same, and the animal will resist a subsequent peritoneal inoculation of a scraped agar culture of the bacillus pyocyaneus.

(4) A fortiori a subcutaneous or intraperitoneal injection of an emulsion of an agar culture of the bacillus pyocyaneus, sterilised or living, will immunise a guinea-pig or rabbit against a subsequent similar intraperitoneal injection of an agar culture of the bacillus pyocyaneus.

(5) This, then, is in full accordance with Klein's experiences, and requires no further comment. Now, if it be a question merely of one " intracellular" poison against another "intracellular" poison, a guinea-pig or rabbit immunised by the above methods might be expected to succumb to an inoculation with old but living bouillon cultures. It was found, however, that such animals resisted large doses of such cultures, which killed control animals in eighteen hours.

(6) Rats are insusceptible to anthrax, but extremely susceptible to an infection with the bacillus pyocyaneus. It was found that our white rats would resist enormous doses of anthrax cultures (eighteen hours old) scraped off agar agar, and made into an emulsion, while they would die after even small doses of similar emulsions prepared with bacillus pyocyaneus, whether they had previously been treated with intraperitoneal injections of anthrax emulsions or not. From these results.we must conclude that anthrax grown on agar agar contains no "intracellular" poison, or that if it does its "intracellular" poison does not immunise against that of the bacillus pyocyaneus. It should be mentioned; however, that though the anthrax cultures used were only eighteen hours old, there were already spores in many of the bacilli, although most of the bacilli were quite free from spores.

There seems to be some special relation between the socalled "intracellular" poison and the pathogenic property of a bacillus, or, differently expressed, if a bacillus is pathogenic to a certain species of animals, then its "intracellular" poison is also pathogenic. Again, if the "intracellular" poison be the same for various species of bacilli, then the lethal doses should be identical for the various bacterial species. Now, it is well known that rabbits are comparatively resistant against cholera infection, and we found that, in spite of injecting large doses of Haffkine's virus fort (a whole tube) intraperitoneally into even young animals, they were none the worse after it. although one-twelfth of a culture of cholera bacilli would kill a guinea-pig. On the other hand, one-fifth of a culture of bacillus pyocyaneus intraperitoneally injected was invariably fatal to rabbits. Again, it required much larger doses of " prodigiosus emul- 
sion" than of "pyocyaneus emulsion" to kill rabbits; the agar cultures least. And also in the case of guinea-pigs "pyocyaneus emulsion" was found to be more virulent than "prodigiosus emulsion." It appears, therefore, that there is something besides the "intracellular" poison which decides the fatal issue. In fact it seems that it is the active metabolism of the bacillus by which the specific toxic substances are produced in the body of the animal. ${ }^{15}$ Why is it that the one bacillus should protect an animal against another or several others, we do not attempt to explain. It may be a purely local immunity. How important the local predisposition and resistance are we know from various experiments, amongst which we may refer to those of Blagovestchensky ${ }^{16}$ and Roger. ${ }^{17}$

(7) While testing this question still further, we immunised guinea-pigs by Haffkine's method, and found that subsequently they resisted intraperitoneal injections of large doses of bouillon and even liquefied gelatine cultures of comma bacilli. So far as gelatine cultures are concerned, our results are in direct contradiction to Klein's. Our gelatine cultures were two months old, and it is possible that they did not contain so much toxin as Klein's. Large doses were used, however, half a culture for each inoculation, which killed the controls in less than twelve hours. If we are justified in assuming that bouillon and liquefied cultures contain the specific cholera toxin, from our experiments it would seem that previous inoculations of non-lethal doses of agar culture emulsions immunise also against the comma bacillus plus its metabolic products. Moreover, we found that the converse holds good, namely, that,

(8) By means of subcutaneous injections of small doses of virulent bouillon cultures of comma bacilli, guinea-pigs are immunised against intraperitoneal injections of large doses of Haffikine's virus fort. We must conclude, therefore, that, after a typical infection short of death, the animal will easily resist the "intracellular" poison.

(9) The result was in no way modified when, instead of living bouillon cultures, cultures heated up to $70^{\circ} \mathrm{C}$. for ten to thirty minutes were used for subcutaneous vaccination. Animals vaccinated in this manner comfortably resisted large doses of the virus fort. In most cases the cultures were not filtered; the issue, however, was the same when cultures were employed for protective vaccination which had been deprived of their bacilli by means of filtration through a Hankin's porcelain filter. Guinea-pigs treated with such filtered cultures proved resistant against Haffkine's virus fort. It is, of course, doubtful whether our filtered or heated cultures contained the true cholera toxin. Large doses of such sterilised cultures, which had been grown under ordinary aërobic conditions, failed to produce death. Others (van Ermengen, Cantani, Nicati and Rietsch, etc.) have succeeded, by means of filtration through Chamberland's filters, in obtaining more active toxic bodies. Hueppe ${ }^{10}$ and Scholl ${ }^{19}$ again claim to have separated from cultures in eggs and choleraic dejecta extremely toxic substances. Gruber and Wiener ${ }^{20}$ have thrown considerable doubt on Scholl's results, at least so far as the toxin extracted from eggs inoculated with comma bacilli is concerned, and Hüppe's method of extraction of toxins from choleraic dejecta is naturally open to criticism. The whole toxicology of cholera is at present in a chaotic condition, and as yet we know but little of the nature of cholera toxins, and so long as the results of various investigators are so contradictory criticism is impossible. We have assumed that our bouillon cultures contain the metabolic products of the cholera bacillus. It is worthy of note, however, that even 5 c.c. of such cultures sterilised by heat injected intraperitoneally failed to produce death. Gruber and Wiener ${ }^{21}$ similarly found that the sterilised peritoneal exudation of animals which had succumbed to intraperitoneal injections of cholera bacilli, even when injected in comparatively large doses, had no fatal effect. while equal or smaller doses of the nonsterilised exudation produced death at least at the first transmission. ${ }^{22}$

That a protection against an infection with comma bacilli can be effected by inoculation with sterilised or filtered bouillon cultures has already been shown by others, notably by Vincenzi, ${ }^{23}$ Gamaleïa, ${ }^{24}$ and Klemperer, ${ }^{25}$ but we have further shown that this immunity holds good also against the inoculation of the intracellular poison or Haffkine's virus fort. ${ }^{26}$

All these experiments throw some doubt on the too narrow or distinctive conception of an intracellular poison, and it seems that within certain limits the virulence of the poison varies with the pathogenic property of the bacillusthat is, with the power of its metabolic toxin. Immunity against bouillon cultures means also immunity against agaragar cultures-that is, the "intracellular" poison and vice vers $\hat{a}$; and immunity against the latter implies immunity against gelatine cultures, unless, perhaps, these cultures contain an excessive dose of toxin. Immunity produced by any one method employed by us-that is, Haffkine's method or preventive inoculation with bouillon cultures, living or sterile, filtered or not-amounts to immunity against any other form of inoculation, at least so long as we are dealing. with the same bacillus.

It is no doubt difficult to explain why an intraperitoneal or subcutaneous injection of "prodigiosus agar emulsion" should protect rabbits or guinea-pigs against an intraperitoneal injection of pyocyaneus agar emulsion, but that it is hardly a question of one intracellular poison against another seems probable from the fact that it is possible, by means of intraperitoneal injections of old bouillon cultures of the bacillus prodigiosus to render rabbits immune against intraperitoneal injections of "pyocyaneus agar emulsion." The whole matter requires further study on broader lines, and this must be left for future investigation.

(10) The following observations also compel us to regard a too dogmatic or systematic distinction between "intracellular" and metabolic poisons in their relation to immunity with some doubt. Guinea-pigs were immunised against. "cholera" by various methods, and their serum used to immunise other guinea-pigs. ${ }^{27}$ It was found that (1) the serum. of an animal immunised by Haffkine's method will readily immunise another guinea-pig against Haffkine's virus fort ; (2) it will also immunise against intraperitoneal injections of large doses of virulent bouillon cultures. Similarly it was found that the serum of one of us inoculated by Haffkine's method will immunise guinea-pigs against intraperitoneal injections of Haffkine's virus fort or virulent bouillon cultures in large doses. It may here be mentioned that it is quite immaterial whether the serum be injected subcutaneously or intraperitoneally, and that large doses of serum ( 4 to 6 c.c.) often caused severe and extensive ulceration of the abdominal wall when injected subcutaneously. In one case a copious intraperitoneal injection caused death in two days, the animal suffering from the usual symptoms of a subacute intoxication. In all cases, whether our own serum or that of immunised guinea-pigs was used, large doses of serum (2 c.c. and more) caused the animals to be ill and feverish for some time (three to four days). There was loss of appetite, and the coat was rough, and the animals hid themselves in the straw. It is quite possible, therefore, that the immunising serum contains some active, if not the specific toxic substance.

(11) It was further found that the serum of guinea-pigs immunised by repeated subcutaneous injections of bouillon cultures heated to $65^{\circ}$ or $100^{\circ} \mathrm{C}$. will immunise others against intraperitoneal injections of large doses of Haffkine's virus fort or virulent bouillon cultures.

One of us was treated by subcutaneous injections of virulent bouillon cultures, while at the same time large doses of sterilised bouillon cultures were administered per os, the gastric secretion having previously been neutralised with a solution of carbonate of sodium. In this case also the serum immunised guinea-pigs against Haffkine's virus fort'. Unfortunately, we omitted to test the immunising power of our own sera before the auto-inoculations. Klemperer ${ }^{28}$ has shown that the serum of some normal individuals has a weak immunising action. It is, however, very slight compared with that of the serum after treatment. Now in our case the immunising power was well marked, since 0.25 c.c. readily immunised guinea-pigs against large doses of Haffkine's virus fort. The serum of normal guinea-pigs, so far as our own experience goes, has no immunising action whatever.

(12) It should also be mentioned that one of us, having been inoculated with repeated subcutaneous injections of virulent bouillon cultures, subsequently, when his serum 
possessed marked immunising properties, showed only slight local reaction against a large dose of Haffkine's virus fort (half tube). This, again, tends to show that the view of the distinct kinds of poisons should not be taken too narrow.

The above-mentioned serum experiments are possibly of some importance if Klemperer's law of the specificity of immunising serum holds good for all cases. The Klemperers insist on the truth of this law, and Behring ${ }^{29}$ agrees with them. According to this law the serum of an animal immunised by inoculations with the bacillus prodigiosus would immunise against this bacillus only, and not against any other infection ; and similarly the serum of an animal immunised against "cholera" should not immunise against an infection with the bacillus prodigiosus. This question has also been touched upon; it will, however, require further careful and critical examination, because fallacies may easily be introduced by reason of the fact that the sera also of naturally immune animals have more or less marked immunising properties. $^{30}$

We have found, in accordance with Klemperer's law, that the serum of one of us treated by Haffkine's method will not protect guinea-pigs against intraperitoneal injections of "prodigiosus and pyocyaneus agar emulsions." Sto far too few experiments have been performed, but if our results be confirmed and Klemperer's experiments with the serum of cholera patients taken into consideration, ${ }^{31}$ it would bring us a step nearer the elucidation of the etiology of Asiatic cholera. Judging from Klein's theory of the intracellular poison and from his unequivocal results, we might have expected that the serum obtained from a person treated by Haffkine's method would protect animals against intraperitoneal injections of both "prodigiosus and pyocyaneus agar emulsions," but it was found that such serum will protect against any form of intraperitoneal injection of cholera bacilli only.

Without laying undue stress on the experiments just mentioned, we are led to the following conclusions after a survey of our own observations and those of others. As far as the cholera bacillus is concerned, (1) any one mode of immunisation will protect an animal against an infection by any other form of inoculation used; (2) the serum of an animal immunised by any one method also protects guinea-pigs against an infection by any one of the various forms of inoculation mentioned; (3) the distinction between an "intracellular" and a "metabolic" poison in their relation to artificial immunity must not be made too narrow.

We do not deny that an actual distinction between the metabolic and the protoplasmic poisons exists. Buchner's "proteins" apparently are what Klein calls the intracellular poison, and it is well known, from the researches of Buchner, Römer, and others, that the "proteins" produce reactions often different from those produced by the bacilli and their metabolic substances. Here, also, we must not be too exclusive. Whether Koch's tuberculin belongs to the proteins, as Buchner asserts, seems somewhat doubtful after the careful chemical studies of Hunter, ${ }^{32}$ but, on the other hand, it is almost certain that it does not contain the specific toxin, at least not in a potent form. Now Vissmann ${ }^{33}$ has shown that after injecting dead tubercle bacilli scraped off agar agar into the ear vein of rabbits typical tubercles appear in the lungs and liver, which, however, show no inclination towards caseation, but rather towards cicatrisation. Unfortunately, Vissmann has omitted to investigate the influence of such intravenous injections on immunity. If, however as Klein and Buchner assume, tuberculin be the intracellular poison, then Kitasato ${ }^{34}$ has shown that by inoculations with this poison it is possible to protect guinea-pigs against a tuberculous infection-a result which confirms our own conclusion that the "intracellular" poison protects against the bacillus and its specific toxin.

\section{REFERENCES.}

1 British' Medical Journal, March '25th, 1893, p. 632, et seq. 2 Zeitschr. f. Hygiene, vol. xi, p. 393 . 3 Semaine Médicale, $1892, \mathrm{pp}$. 285, 293, and 311 ; see f. Hygiene, vol. xi, p. 393. 3 Semaine Medicale, 1892, pp. 285, 293, and 311 ; see
also paper by A. E. Wright and D. Bruce, BRITISH MEDICAL JOURNA, also paper by A. E. Wright and D. Bruce, BRITISH MEDICAL JouRNAL,

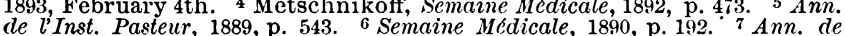
l'Inst. Pasteur. 1890, p. 545. ${ }^{\circ}$ Ann de l'Inst. Pasteur, May, 1992. 9 Ann. de ${ }^{\prime}$ 'Inst. Pasteur, March, 1893, p. 267. 10 Klein, loc. cit.; and Gruber and Wiener, Archiv f. Hygiene, $\mathrm{xv}, \mathrm{p} .263 .11$ Op. cit. ${ }_{12}$ Op. cit. p. 261. ${ }_{13}$ Op. cit. ${ }^{14}$ Op. cit., p.266. ${ }_{15} \mathrm{Cf}$., also Gruber and Wiener, op. cit., p. 266.
16 Ann. de l'Inst.1 Pasteur, 1890, ii. ${ }^{17}$ Rev. de Méd., 1891, pp. 169 and 500. 18 Prager med. Wochenschr., 1890, No. 44, and Berl. klin. Wochenschr., 1893. 19 Archiv f. Hygiene 1892 , p. 208. 20 Op. cit., p. 302.21 Op cit., pp. 297 and 298. 22 Op.cit., p. 288. 23 Deutsche med. Wochenschr., 1892, May 5th and 1893. 24 Acad. des Sciences, 1888, Aug. 20th and Compt. Rend. de la Soc. de Biol., 1889, 38. 3erl. klin. Wochenschr., 1892, August 8th. 26 Gruber and Wiener, op. cit., pp. 302 to 313 . 27 Vincenzi, loc. cit.; Klemperer, loc. cit. 28 Op. cit. 29 Die Blutserum Therapie, Die Geschichte der Diphtherie, and Deutsche med. Wochen-

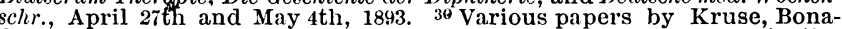
duce, Pansine, Ziegler's Beitrage, xii, 3 , and by the two Klemperers in the Berl. klin. Wochenschr., 1891 and 1892. 31 Berl. klin. Wochenschr., August 8th, 1892. 32 British MediCAL JourNAL, 1891. 33 Virchow's Archiv, 129, pp. 163 to 170.34 Zeitschr. f. Hygiene, 1892.

\section{THE PRESENT POSITION OF THE SURGERY OF THE HYPERTROPHIED PROSTATE.}

Abstract of an Address delivered before the American Surgical Association.

By J. WILLIAM WHI'TE, M.D.,

Professor of Clinical Surgery, University of Pennsylvania; Surgeon to the University, German, and Philadelphia Hospitals.

Professor White alluded to the admirable papers in which Harrison, ${ }^{1}$ McGill, ${ }^{2}$ Belfield ${ }^{3}$ and Moullin ${ }^{4}$ had so thoroughly reviewed this subject, and acknowledged his indebtedness to them for the basis of the address. ${ }^{5}$ The main practical points in reference to enlargement of the prostate are to be classified as follows: 1 . The nature and chief varieties of the prostatic enlargement and their relation to the vesical changes found associated with them. 2. The symptoms in relation to diagnosis and prognosis, (a) subjective, (b) objective. 3. The indications for $(a)$ non-interference, $(b)$ medical treatment, (c) palliative treatment, (d) operative treatment. 4. The choice of operation. 5. The methods of operation.

1. As to the nature of the enlargement, he rejects the view which assigns prostatic hypertrophy to the class of disseminated arterial scleroses (Guyon); and that which makes it secondary to vesical changes (Harrison), and thinks we are warranted in believing the function of the gland to be chiefly sexual, and in provisionally accepting the theory which recognises that the growth or growths which make up the enlargement in prostatic hypertrophy are analogous to those fibromyomata so frequently found in the uterus. This, if true, could scarcely be said to be a full explanation of their occurrence, but by allying them to a well-known group of tumours would certainly bring us a step nearer to their comprehension. The importance of the conclusion lies in its effect upon prognosis and treatment. If the condition is merely a part of a general or constitutional state, or if it is secondary to changes in the vesical walls themselves, it is obvious that comparatively little is to be expected from treatment, operative or otherwise. If, on the other hand, it is a local condition, dependent on causes at present unknown, but which may be said to be inherent in the structure or in the functional condition of the gland itself, and if the changes in the bladder walls and the accumulation of residual urine are the result of the precedent prostatic disease, treatment of the latter, if early and judicious, offers much more prospect of success.

The changes in the bladder are occasioned by (1) the mechanical obstruction which the enlarged prostate offers to the ready and complete evacuation of its contents, (2) by the circulatory disturbance incident to pressure on the prostatic veins into which the blood from the vesical veins passes, and (3) to septic infection. Septic infection of a healthy mucous membrane by the pyogenic microbes of acute or chronic cystitis is not possible, even though such bacteria are present in the urine; when, however, the vesical mucous membrane is congested in consequence of obstruction to venous return, and by distension and frequently recurring contractions of the detrusor muscles, it offers but slight resistance to the microbic invasion. The pyogenic microbes are generally carried to the bladder by dirty instruments, or, if these are rendered sterile, through failure to cleanse the anterior urethra before the instrument is introduced into the bladder. Often cystitis develops independently of the use of instruments, probably as a result of infection conveyed by way of the urethral mucous membrane. 